\title{
MicroRNA-7 inhibits colorectal cancer cell proliferation, migration and invasion via TYRO3 and phosphoinositide 3-kinase/protein B kinase/mammalian target of rapamycin pathway suppression
}

\author{
ANCHENG QIN and WEIFENG QIAN \\ Department of General Surgery, The Affiliated Suzhou Hospital of Nanjing Medical University, \\ Suzhou, Jiangsu 215002, P.R. China
}

Received April 20, 2018; Accepted August 29, 2018

DOI: $10.3892 /$ ijmm.2018.3864

\begin{abstract}
MicroRNA-7 (miR-7) is a non-coding RNA that inhibits colorectal cancer (CRC) cell proliferation, migration and invasion. miR-7 effectively silences TYRO3 expression, and the close association between TYRO3 and CRC has previously been reported. Therefore, the present study aimed to assess the roles and molecular mechanisms of TYRO3 and miR-7 in the development of CRC. The expression levels of miR-7 and TYRO3 in CRC tissues and cell lines were detected by reverse transcription-quantitative polymerase chain reaction. A dual-luciferase reporter assay was also performed to confirm whether TYRO3 was a target of miR-7. The effect of miR-7 and TYRO3 on cell proliferation, migration and invasion was detected in vitro with MTT, wound healing and cell invasion assays, respectively. The expression of proteins associated with the phosphoinositide 3-kinase/protein kinase $\mathrm{B} / \mathrm{mammalian}$ target of rapamycin (PI3K/AKT/mTOR) pathway was detected by western blot analysis. The results indicated that the expression of miR-7 was downregulated and the levels of TYRO3 were gradually increased in CRC tissues and cell lines. TYRO3 was identified as a functional target of miR-7, and its expression was negatively regulated by miR-7 in CRC cell lines. The functional studies demonstrated that miR-7 knockdown promoted the proliferation, migration and invasion of CRC cells, while knockdown of TYRO3 repressed these biological processes. In addition, pathway analyses revealed that the oncogenic effect of TYRO3 was associated with PI3K/AKT/mTOR pathway inhibition. In conclusion, the data suggested that miR-7 promoted the development of CRC
\end{abstract}

Correspondence to: Dr Weifeng Qian, Department of General Surgery, The Affiliated Suzhou Hospital of Nanjing Medical University, 26 Dao-qian Road, Suzhou, Jiangsu 215002, P.R. China E-mail: qianwf163@163.com

Key words: microRNA-7, TYRO3, colorectal cancer, proliferation, migration, invasion, phosphoinositide 3-kinase/protein kinase $\mathrm{B} /$ mammalian target of rapamycin pathway by targeting oncogenic TYRO3, which may be mediated by inhibition of the PI3K/AKT/mTOR signaling pathway. Thus, miR-7 may serve as an independent prognostic biomarker in patients with CRC.

\section{Introduction}

Colorectal cancer (CRC) is one of the most common malignancies worldwide and the third leading cause of cancer-associated mortality (1). According to the statistics, there are 1.36 million new cases of CRC and 694,000 CRC-associated mortalities annually worldwide (2). Cancer cell metastasis is one of the major problems that hinders successful CRC treatment $(3,4)$. Increasing evidence indicated that cell proliferation, migration and invasion are crucial in CRC metastasis. However, the potential molecular mechanism underlying CRC metastasis has yet to be fully elucidated. Therefore, investigating the molecular mechanisms involved in CRC progression is crucial in order to develop novel and effective therapies.

MicroRNAs (miRNAs/miRs) are a type of highly conserved small non-coding RNA with a length of approximately 19-25 nucleotides. miRNA post-transcriptionally regulates the expression of a target gene through direct interaction with the 3'-untranslated region (3'UTR) of its target mRNA, and is known to be involved in cell proliferation, migration and invasion (5). To date, miRNAs have emerged as potential critical regulators of carcinogenesis and tumor progression $(6,7)$. A variety of miRNAs have been reported to serve as anti-oncogenes in CRC, including miR-483 and miR-551 (8). miR-7 is an evolutionarily conserved miRNA that is involved in the development of the eye and pancreas in Drosophila (9). Accumulating evidence suggests that miR-7 simultaneously targets a number of mRNAs that are involved in various signaling pathways in several types of cancer (9). It has been demonstrated that miR-7 is downregulated in certain human tumors, including $\mathrm{CRC}$, and this miRNA has been reported to regulate a number of oncogenic signal transduction pathways, including the epidermal growth factor receptor signaling pathway, as well as the phosphoinositide 3-kinase/protein kinase B (PI3K/AKT) and RAF/mitogenactivated protein kinase kinase/extracellular signal-regulated 
kinase (RAF/MEK/ERK) pathways, suggesting that it may function as a tumor suppressor $(10,11)$. The present study aimed to examine the role of miR-7 in CRC and identify novel targets that may be clinically useful.

TYRO3, a member of the TAM family (comprising TYRO3, AXL and MERTK) of tyrosine kinases (12), has been demonstrated to be abnormally expressed in a wide variety of human tumors, including CRC, and to be associated with tumor progression and resistance to targeted therapeutics $(13,14)$. It has been reported that the receptor tyrosine kinase Axl promotes cell migration and invasion in CRC (15). In addition, overexpression of TYRO3 in tumor tissues significantly reduced the survival of patients with CRC (13). TYRO3, as a novel functional target of miR-7, was reported to regulate the proliferation, invasion and migration of Huh-7 cells via the PI3K/AKT pathway (16). This signaling pathway serves a key role in several cancer processes, including proliferation, tumor growth and tumorigenesis $(17,18)$. A previous study has reported that abnormal activation of PI3K/AKT promotes the invasion and metastasis of numerous tumors, including CRC (18). However, whether miR-7 regulates cell proliferation, invasion and migration in CRC via TYRO3 and subsequent PI3K/AKT pathway inhibition has not been elucidated to date.

In the present study, aimed to investigate the role of miR-7 in CRC and its potential mechanism. The results demonstrated that miR-7 was significantly downregulated in CRC. Overexpression of miR-7 inhibited the proliferation, migration and invasion of CRC cell lines by directly inhibiting the TYRO3 receptor tyrosine kinase, resulting in the inhibition of PI3K/AKT pathway, with a significant impact on cancer cell migration, proliferation and invasion.

\section{Materials and methods}

Patients. A total of $30 \mathrm{CRC}$ tissue samples and corresponding matched adjacent noncancerous tissues were obtained from patients with CRC at the Affiliated Suzhou Hospital of Nanjing Medical University (Suzhou, China) between March 2014 and September 2017. The mean age of the included patients was $63 \pm 8$ years and the age range was $34-74$ years. The diagnosis of CRC was histologically confirmed in all patients based on colonoscopy findings (19). No patients had received chemotherapy or radiation therapy. Each patient provided written informed consent, and all experimental protocols were approved by the Ethics Committee of the Affiliated Suzhou Hospital of Nanjing Medical University (Suzhou, China).

Cell lines and culture. The human colon cancer cell lines LoVo, SW480, SW620, HCT116 and HT29, as well as 293 cells, were purchased from the American Type Culture Collection (Manassas, VA, USA). The normal human colonic epithelium cell line NCM460 was purchased from the Cell Bank of the Chinese Academy of Sciences (Shanghai, China). LoVo, SW480, SW620, HCT116 and HT29 cells were cultured in RPMI-1640 medium (Gibco; Thermo Fisher Scientific, Inc., Waltham, MA, USA) supplemented with $10 \%$ fetal bovine serum (FBS; Gibco; Thermo Fisher Scientific, Inc.), $100 \mathrm{U} / \mathrm{ml}$ penicillin and $100 \mathrm{mg} / \mathrm{ml}$ streptomycin in a humidified atmosphere with $5 \% \mathrm{CO}_{2}$ at $37^{\circ} \mathrm{C}$. NCM460 and 293 cells were cultured in Dulbecco's modified Eagle's medium (Gibco;
Thermo Fisher Scientific, Inc.) supplemented with 10\% FBS, $100 \mathrm{U} / \mathrm{ml}$ penicillin and $100 \mathrm{mg} / \mathrm{ml}$ streptomycin at $37^{\circ} \mathrm{C}$ in a $5 \% \mathrm{CO}_{2}$ incubator.

Reagents. The miR-7 mimic, miR-7 inhibitor, TYRO3 small interfering RNA (siRNA) (cat. no. stB0004873C-1-5) and the negative controls (NC) were purchased from Guangzhou RiboBio Co., Ltd. (Guangzhou, China). The detailed information regarding miR-7 mimic, miR-7 inhibitor, siRNA and their controls is as follows: i) miR-7 mimic sense, 5'-UGGAAGACU AGUGAUUUUGUUGU-3' and antisense, 5'-AACAAAAUC ACUAGUCUUCCAUU-3'; NC of miR-7 mimic sense, 5'-UUC UCCGAACGUGUCACGUTT-3' and antisense, 5'-ACGUGA CACGUUCGGAGAATT-3'; ii) miR-7 inhibitor, 5'-ACA ACAAAAUCACUAGUCUUCCA-3'; NC of miR-7 inhibitor, 5'-CAGUACUUUUGUGUAGUACAA-3'; iii) TYRO3 siRNA sense, 5'-GAGCUUUACUUGUCUGCGATT-3' and antisense, 5'-UCGCAGACAAGUAAAGCUCGG-3'. The pmiR-REPORT-TYRO3 3'UTR wild-type (WT) and the pmiR-REPORT-TYRO3 3'UTR mutant (MUT) sequences were synthesized by GenScript, Inc. (Piscataway, NJ, USA).

Transfections. LoVo, SW480 and SW620 cells were seeded in 6-well plates at a concentration of (1-1.5)x $10^{5}$ cells/well (25-35\% confluence) for $48 \mathrm{~h}$ before transfection. Subsequently, Lipofectamine 2000 reagent (Invitrogen; Thermo Fisher Scientific, Inc.) was used for transient transfection of cells with miR-7 mimic $(50 \mathrm{nM})$, miR-7 inhibitor $(100 \mathrm{nM})$ or NC (100 nM), while Oligofectamine transfection reagent (Thermo Fisher Scientific, Inc.) was used for transfection with TYRO3 siRNA (200 nmol/l) or transfection of cells with miR-7 inhibitor + TYRO3 siRNA for $48 \mathrm{~h}$, following the manufacturer's protocol.

Dual-luciferase reporter assay. The prediction programs TargetScan (http://www.targetscan.org/vert_72/), PicTar (http://www.pictar.org) and miRanda (http://www.microrna. org) were used to predict the potential targets of miR-7. Luciferase assays were conducted using 293 cells, which were seeded in 24-well plates at $2 \times 10^{4}$ cells/well and incubated overnight. The pmiR-REPORT-TYRO3 3'UTR WT or MUT luciferase plasmid (500 ng) along with $30 \mathrm{nM}$ miR-7 mimic or miR-NC was co-transfected into 293 cells using Lipofectamine 2000 reagent. Subsequent to transfection for $48 \mathrm{~h}$, cells were harvested, and a Dual-Luciferase Reporter Assay kit (Promega Corporation, Madison, WI, USA) was used to measure the luciferase activity according to the manufacturer's protocol. Luciferase activity was normalized to Renilla luciferase activity.

Reverse transcription-quantitative polymerase chain reaction $(R T-q P C R)$. Total RNA was extracted from the tissues and cells using TRIzol reagent (Invitrogen; Thermo Fisher Scientific, Inc.) and an miRNeasy extraction kit (Qiagen, Inc., Valencia, CA, USA), according to the manufacturer's protocol. The RNA concentration was qualitied by Uv-vis at $280 \mathrm{~nm}$. Single-stranded cDNA was synthesized with a QuantiTect Reverse Transcription kit (Qiagen, Inc.). Next, qPCR was performed using SYBR-Green Master Mix (Applied Biosystems; Thermo Fisher Scientific, Inc.) in an ABI 7500 
thermocycler (Applied Biosystems; Thermo Fisher Scientific, Inc.), and the results were normalized to GAPDH expression. The following primer sequences were used: TYRO3 sense, 5'-GTGTGTGGCTGACTTCGGAC-3', and antisense, 5'-CAC GTCCTCCATACACTCCG-3'; GAPDH sense, 5'-GGAGCC AAAAGGGTCATCAT-3', and antisense, 5'-GTGATGGCA TGGACTGTGGT-3'. The reaction conditions were conducted as follows: 40 cycles of predenaturation for $10 \mathrm{~min}$ at $95^{\circ} \mathrm{C}$, denaturation for $30 \mathrm{sec}$ at $95^{\circ} \mathrm{C}$, annealing for $20 \mathrm{sec}$ at $60^{\circ} \mathrm{C}$ and extension for $35 \mathrm{sec}$ at $72^{\circ} \mathrm{C}$. The expression of mature miR-7 was quantified with a TaqMan microRNA assay kit (Applied Biosystems; Thermo Fisher Scientific, Inc.) and was detected relative to U6 small nuclear RNA expression. The relative expression of each gene was calculated using the $2^{-\Delta \Delta \mathrm{Cq}}$ method (20).

Cellproliferation assay. Cell proliferation was detected using an MTT assay [also known as 3-(4,5-dimethyl-2-thiazolyl)-2,5-diphenyl-2-H-tetrazolium bromide; Sigma-Aldrich; Merck KGaA, Darmstadt, Germany] following transfection with miR-7 mimic, miR-7-inhibitor, NC and TYRO3 siRNA. At $48 \mathrm{~h}$ post-transfection, the LoVo, SW480 and SW620 cells $\left(3 \times 10^{3}\right.$ cells/well) were seeded in 96 -well culture plates for $24 \mathrm{~h}$, and then incubated with $20 \mu \mathrm{l}$ MTT $(5 \mathrm{mg} / \mathrm{ml})$ for $4 \mathrm{~h}$ at $37^{\circ} \mathrm{C}$. Next, the culture medium was removed and dimethyl sulfoxide (150 $\mu$; ; Sigma-Aldrich; Merck KGaA) was added into each well for $30 \mathrm{~min}$ until all crystals had been dissolved. Absorbance was measured at $570 \mathrm{~nm}$ using a spectrophotometer (Ultrospec 2000; GE Healthcare Life Sciences, Little Chalfont, UK).

Wound healing assay. A wound healing assay was conducted to measure cell migration. Briefly, LoVo, SW480 and SW620 cells $\left(3 \times 10^{5}\right.$ cells/well) were seeded into 6-well plates. After $48 \mathrm{~h}$ of transfection, a $10-\mu 1$ sterile pipette tip was used to scrape the cell monolayer. The migration path of cells was subsequently tracked at 0 and $24 \mathrm{~h}$ after wounding using a phase contrast microscope (IX711; Olympus Corporation, Tokyo, Japan). Quantitative analysis of the wound healing area was performed using ImageJ software (National Institutes of Health, Bethesda, MA, USA).

Cell invasion assay. The cell invasion assay was performed in 24-well chambers containing a Transwell membrane filter (Corning Incorporated, Corning, NY, USA). After 48-h transfection, LoVo, SW480 and SW620 cells were resuspended in $200 \mu \mathrm{l}$ serum-free medium and subsequently seeded into the upper chamber of the plates. A total of $600 \mu \mathrm{l}$ medium containing $10 \%$ FBS was placed in the lower chamber. Subsequent to incubation for $24 \mathrm{~h}$, cells on the upper membrane were removed with a cotton swab. The number of cell invading the lower chamber was used to evaluate the invasive capacity, by counting and averaging the cells in five random fields for each well at a magnification of x100.

Western blot analysis. Total protein was extracted from the cells using a protein lysis buffer (Beyotime Institute of Biotechnology, Haimen, China), and the protein concentration was measured with a bicinchoninic acid assay kit (Beyotime Institute of Biotechnology). Protein samples (30 $\mu \mathrm{g}$ each) were then separated by $10 \%$ SDS-PAGE (Sigma-Aldrich; Merck $\mathrm{KGaA}$ ) and transferred onto polyvinylidene difluoride membranes (EMD Millipore, Billerica, MA, USA). The membranes were blocked with $5 \%(\mathrm{w} / \mathrm{v})$ skimmed milk in Tris-buffered saline/Tween-20 for $1 \mathrm{~h}$ at room temperature and subsequently stained overnight at $4^{\circ} \mathrm{C}$ with primary antibodies at a dilution of 1:1,000, as follows: Anti-TYRO3 (cat. no. 5585), anti-phospho-PI3K (Tyr458; cat. no. 4228), anti-PI3K (cat. no. 4292), anti-AKT (cat. no. 9272), anti-phospho-AKT (Ser473; cat. no. 4060), anti-mammalian target of rapamycin (mTOR; cat. no. 2972), anti-phospho-mTOR (Ser2448; cat. no. 2971) and anti-GAPDH (14C10; cat. no. 2118; all purchased from Cell Signaling Technology, Inc., Danvers, MA, USA). Membranes were subsequently incubated with horseradish peroxidase-conjugated anti-rabbit IgG secondary antibody (1:2,000; cat. no. 7074; Cell Signaling Technology, Inc.) for $1 \mathrm{~h}$ at room temperature. Proteins bands were visualized using an enhanced chemiluminescence detection system (GE Healthcare Life Sciences). ImageJ software, version 1.46 (National Institutes of Health, Bethesda, MD, USA) was applied to quantify the integrated density of the bands.

Statistical analysis. All data were analyzed using SPSS software, version 13.0 (SPSS, Inc., Chicago, IL, USA). The difference in miR-7 expression between CRC tissues and adjacent normal tissue samples was determined by paired t-test. Statistical differences between the groups were assessed by one-way analysis of variance, followed by Tukey post-hoc test. The data are presented as the mean \pm standard deviation. $\mathrm{P}<0.05$ was considered to denote a statistically significant difference.

\section{Results}

miR-7 is downregulated in CRC tissues and cell lines. The expression of miR-7 in CRC tissues and CRC cell lines was detected by RT-qPCR. As shown in Fig. 1A, the expression of miR-7 was significantly decreased in CRC tissues as compared with that in adjacent normal tissue samples. In addition, the expression of miR-7 mRNA was markedly downregulated in all five CRC cell lines compared with that in normal colonic mucosa epithelial cells (Fig. 1B). These results indicated that miR-7 may be a potential tumor suppressor and may be involved in the development of CRC.

miR-7 overexpression inhibits the proliferation, migration and invasion of CRC cells. In order to investigate whether miR-7 serves a role in the development and progression of $\mathrm{CRC}$, the LoVo, SW480 and SW620 cell lines were transfected with miR-7 mimic or miR-NC. RT-qPCR analysis demonstrated that miR-7 mimic transfection effectively increased miR-7 mRNA expression (Fig. 2A). The effect of miR-7 overexpression on the migration of $\mathrm{CRC}$ cells was then assessed with a wound healing assay. As shown in Fig. 2B, transfection with miR-7 mimic significantly inhibited the migration of LoVo, SW480 and SW620 cells, as compared with that observed in the control group. MTT and Transwell assays further revealed that miR-7 overexpression significantly inhibited the proliferation and invasion of LoVo, SW480 and SW620 cells compared with the control group (Fig. 3A and B). Taken together, these 
A

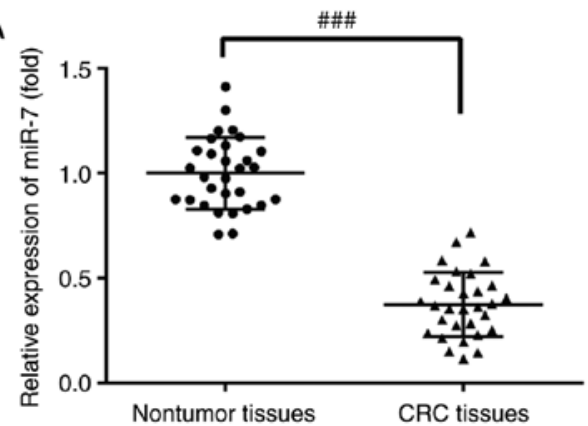

$\mathrm{B}$

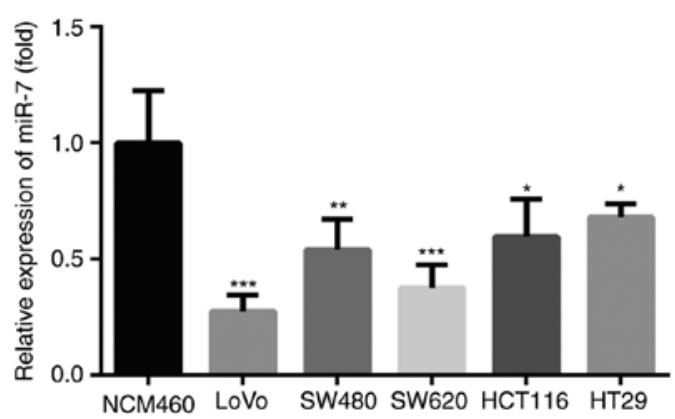

Figure 1. miR-7 was downregulated in CRC tissues and cell lines. The expression of miR-7 in (A) CRC and adjacent normal tissues, and in (B) CRC cells (LoVo, SW480, SW620, HCT116 and HT29) and normal colonic mucosa epithelial NCM460 cells was detected by reverse transcription-quantitative polymerase chain reaction. Data are expressed as the mean \pm standard deviation. ${ }^{\# \# \# ~} \mathrm{P}<0.001$ vs. adjacent normal tissues; ${ }^{*} \mathrm{P}<0.05,{ }^{* * *} \mathrm{P}<0.01$ and ${ }^{* * * *} \mathrm{P}<0.001$, vs. normal colonic mucosa epithelial NCM460 cells. CRC, colorectal cancer; miR-7, microRNA-7.
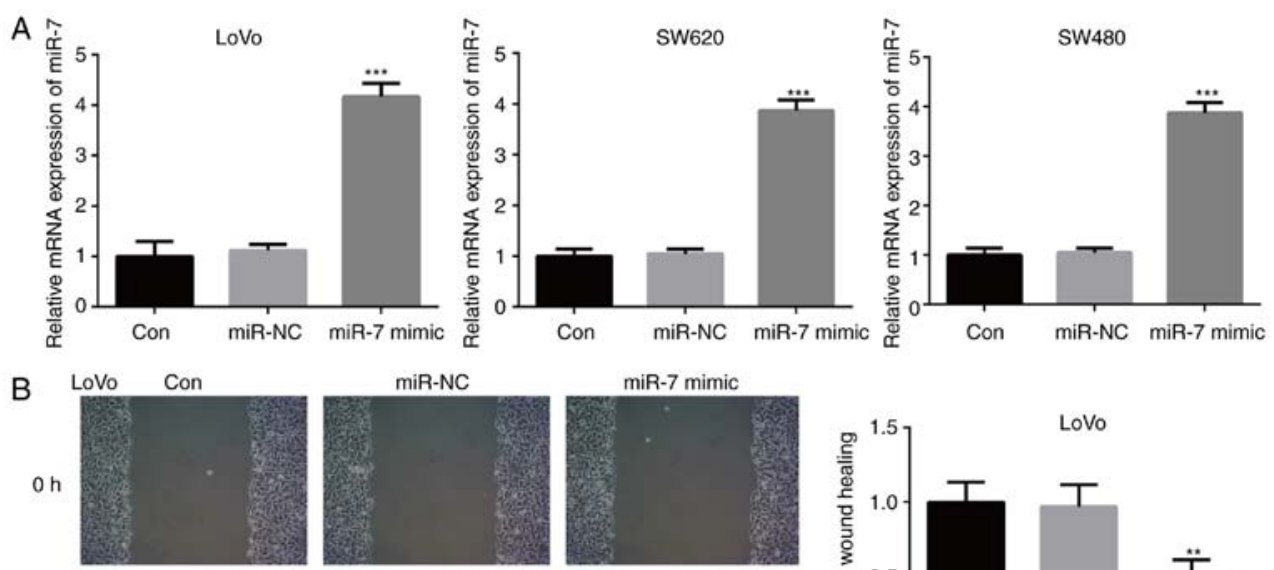

miR-7 mimic
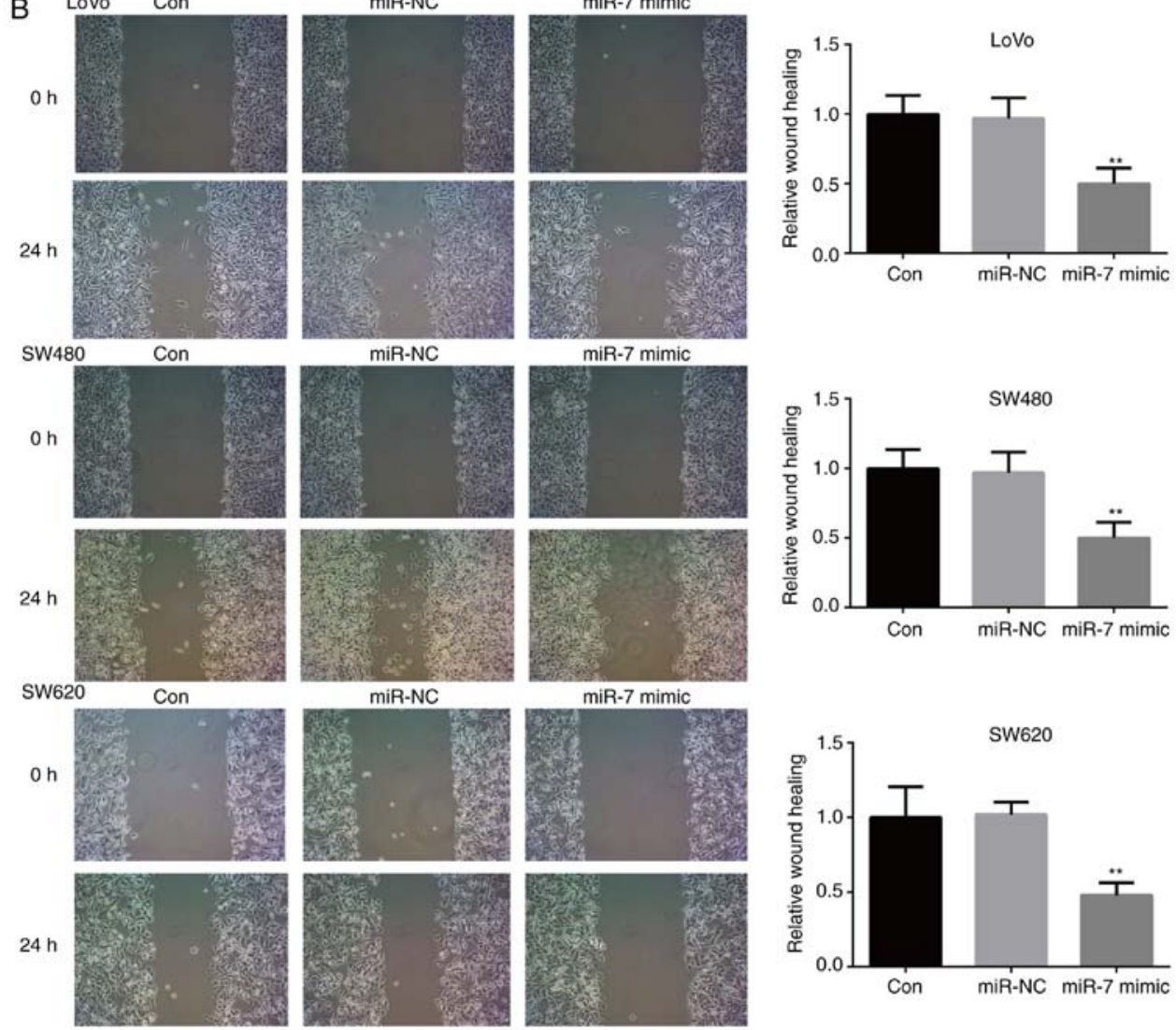

Figure 2. miR-7 overexpression inhibited colorectal cancer cell migration. (A) Reverse transcription-quantitative polymerase chain reaction was used to detect miR-7 expression in LoVo, SW480 and SW620 cells transfected with miR-NC or miR-7 mimic. (B) Effect of miR-7 overexpression on the migration of LoVo, SW480 and SW620 cells was assessed by a wound healing assay. Data are expressed as the mean \pm standard deviation. ${ }^{* *} \mathrm{P}<0.01$ and ${ }^{* * *} \mathrm{P}<0.001$ vs. Con group. miR, microRNA; Con, Control; NC, negative control.

results suggested that miR-7 served an important role in CRC progression.
TYRO3 is upregulated in CRC cells and is a direct target gene of $m i R$-7. As reported earlier, miR-7 was downregulated in 

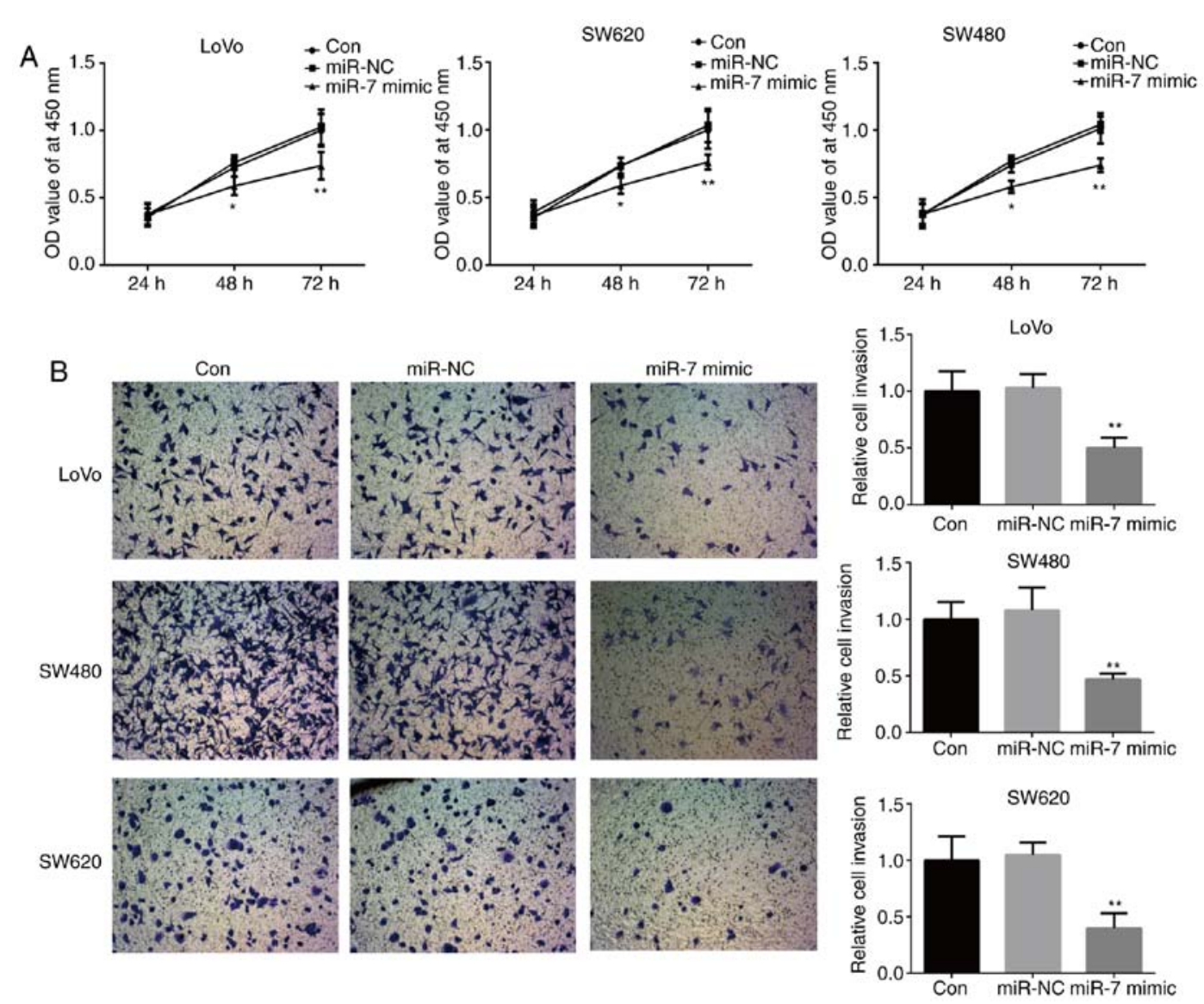

Figure 3. miR-7 overexpression inhibited colorectal cancer cell proliferation and invasion. (A) Effect of miR-7 overexpression on the proliferation of LoVo, SW480 and SW620 cells transfected with miR-NC or miR-7 mimic, assessed by an MTT assay. (B) Effect of miR-7 overexpression on the invasion of LoVo, SW480 and SW620 cells transfected with miR-NC or miR-7 mimic, assessed by a Transwell assay. Data are expressed as the mean \pm standard deviation. ${ }^{*} \mathrm{P}<0.05$ and ${ }^{* *} \mathrm{P}<0.01$ vs. Con group. miR, microRNA; Con, Control; NC, negative control.

CRC tissues and cell lines (Fig. 1). The protein and mRNA expression levels of TYRO3 were subsequently assessed, and the results revealed that these levels were significantly increased in CRC cells compared with those in NCM460 cells (Fig. 4A and B). The prediction programs TargetScan, PicTar and miRanda were used to predict the potential targets of miR-7, and TYRO3 was predicted to be a target gene of miR-7. To further confirm whether TYRO3 was a direct target gene of miR-7, TYRO3 3'UTR WT and MUT were co-transfected into 293 cells along with miR-7 mimic or miR-NC. As presented in Fig. 4C, miR-7 reduced the relative luciferase activity of TYRO3 3'UTR WT, whereas luciferase activity was unaffected in the MUT binding sites. In addition, the effect of miR-7 mimic transfection on TYRO3 expression in LoVo, SW480 and SW620 cells was determined by western blot analysis, and the results indicated that miR-7 overexpression led to a significant decrease in TYRO3 expression in the CRC cells (Fig. 4D).

TYRO3 regulates $C R C$ cell proliferation, migration and invasion via the PI3K/AKT pathway. To evaluate the effects of TYRO3 on CRC, LoVo, SW480 and SW620 cells were transfected with TYRO3 siRNA. Knockdown of TYRO3 significantly inhibited the proliferation, migration and invasion of CRC cells when compared with the control group (Figs. 5A, B and 6). Furthermore, in order to identify the potential mechanism of TYRO3 in the oncogenic processes of CRC cells, the activity of PI3K/AKT/mTOR pathway was detected by western blot analysis. The levels of phosphorylated proteins PI3K, AKT and mTOR were significantly reduced in TYRO3-deficient CRC cells (Fig. 7A-C). These data indicated that the PI3K/AKT/mTOR pathway was involved in the pro-tumorigenic signaling of TYRO3 in CRC cells.

TYRO3 represses the function of miR-7 in CRC cells. As presented in Fig. 8A, the expression of TYRO3 in LoVo, SW480 and SW620 cells transfected with miR-7 inhibitor was significantly increased compared with that in the control group. The effect of co-treatment with TYRO3-siRNA and miR-7 inhibitor was then examined, and co-transfection with TYRO3-siRNA and miR-7 inhibitor effectively reduced TYRO3 expression (Fig. 8A). The results also demonstrated that the proliferation rate of LoVo, SW480 and SW620 cells co-transfected with the miR-7 inhibitor and TYRO3-siRNA was significantly decreased as compared with that in the group treated with miR-7 inhibitor alone (Fig. 8B). Furthermore, the migration and invasion abilities of LoVo, SW480 and SW620 cells co-transfected with the TYRO3-siRNA and miR-7 inhibitor were significantly inhibited compared with the group treated with miR-7 inhibitor alone (Figs. 8C and 9). These results suggested that TYRO3 may repress the effects of miR-7 on CRC cell proliferation, migration and invasion. 

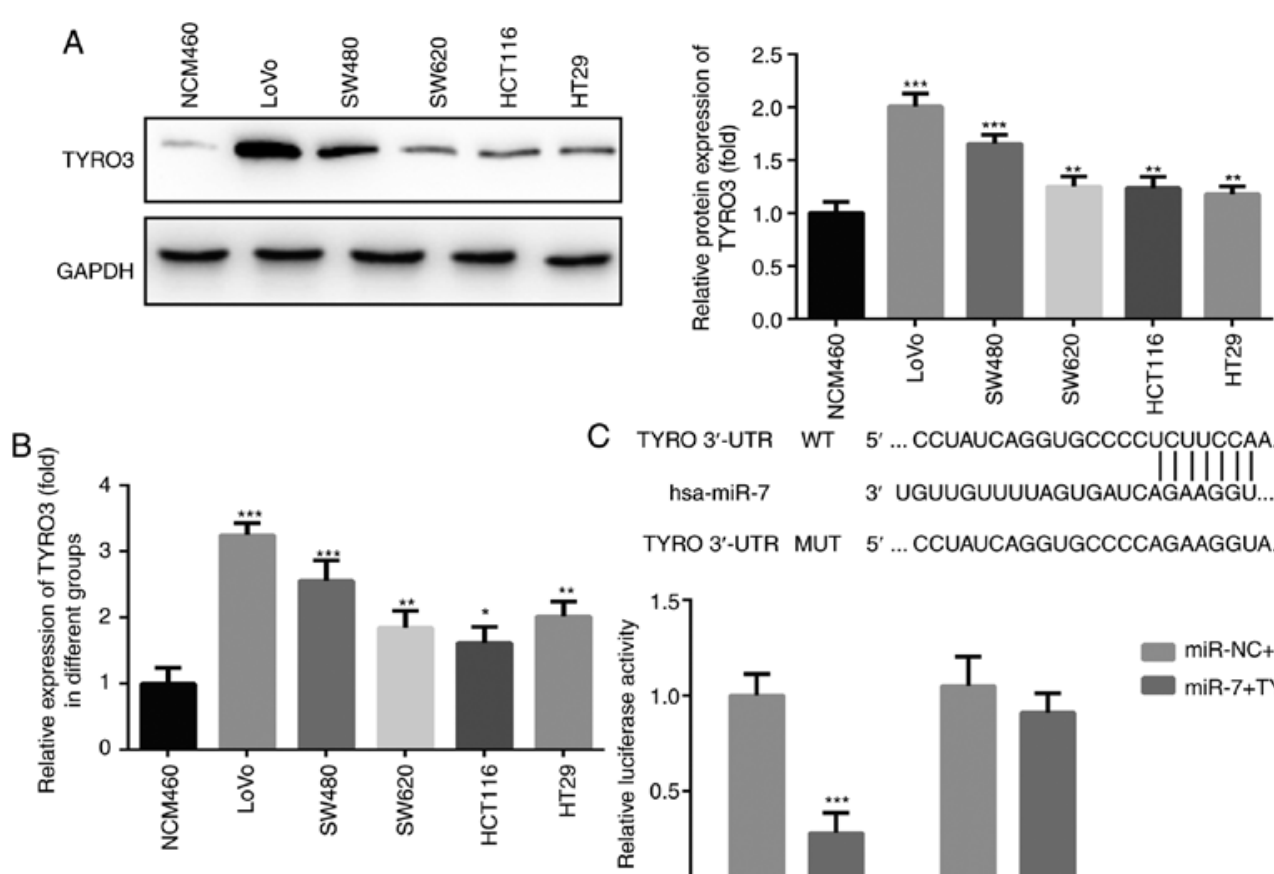

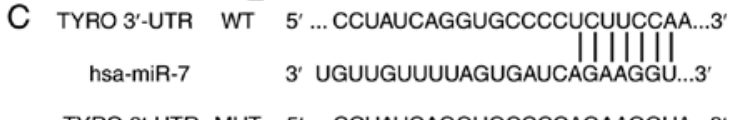

TYRO $3^{\prime}$-UTR MUT $5^{\prime}$... CCUAUCAGGUGCCCCAGAAGGUA...3'


Figure 4. TYRO3 was upregulated in colorectal cancer cells and is a direct target gene of miR-7. (A) Western blot analysis and (B) reverse transcriptionquantitative polymerase chain reaction were used to determine the expression of TYRO3 in LoVo, SW480, SW620, HCT116, HT29 and normal colonic mucosa epithelial cells. (C) Luciferase activity in cells co-transfected with TYRO3 3'UTR WT/MUT and miR-7 mimic/mimic control. (D) Protein expression of TYRO3 in LoVo, SW480 and SW620 cells transfected with miR-7 mimic or miR-NC was detected by western blot analysis. Data are expressed as the mean \pm standard deviation. ${ }^{*} \mathrm{P}<0.05,{ }^{* * *} \mathrm{P}<0.01$ and ${ }^{* * * *} \mathrm{P}<0.001$, vs. corresponding control group. miR, microRNA; Con, Control; NC, negative control; 3'UTR, 3'-untranslated region; WT, wild-type; MUT, mutant.

miR-7 inhibits the activation of PI3K/AKT pathway by directly targeting TYRO3 in CRC cells. In order to understand the molecular mechanism of miR-7 on the biological behavior of CRC cell lines, alterations in the protein expression levels of p-PI3K, PI3K, p-AKT, AKT, p-mTOR and mTOR in LoVo, SW480 and SW620 cells transfected with miR-7 inhibitor or with TYRO3-siRNA plus miR-7 inhibitor were assessed by western blotting. As presented in Fig. 10A-C, compared with the control group, the protein levels of p-PI3K, p-AKT and p-mTOR were significantly increased in LoVo, SW480 and SW620 cells transfected with miR-7 inhibitor, suggesting that the PI3K/AKT pathway was activated. However, this effect was suppressed by co-transfection with si-TYRO3 and miR-7 inhibitor. These results indicated that miR-7 suppressed the activation of the PI3K/AKT/mTOR signaling pathway via direct targeting of TYRO3 in CRC cells.

\section{Discussion}

CRC is one of the most common types of cancer, posing a serious societal and economic burden worldwide. Accumulating evidence has suggested that miRNAs serve an important role in CRC progression. In the present study, it was determined that miR-7 was downregulated in CRC tissues and cell lines. In addition, it was demonstrated that miR-7 inhibited the proliferation, migration and invasion of CRC cells via TYRO3 through the inhibition of the PI3K/AKT/mTOR signaling pathway.

A previous study reported that elevated miR-7 expression was significantly correlated with tumor depth, venous invasion, lymphatic invasion, lymph node metastasis and liver metastasis (21). Previous studies have also reported that miR-7 functions as a tumor suppressor in several types of human cancer, including CRC $(11,22)$. High miR-7 

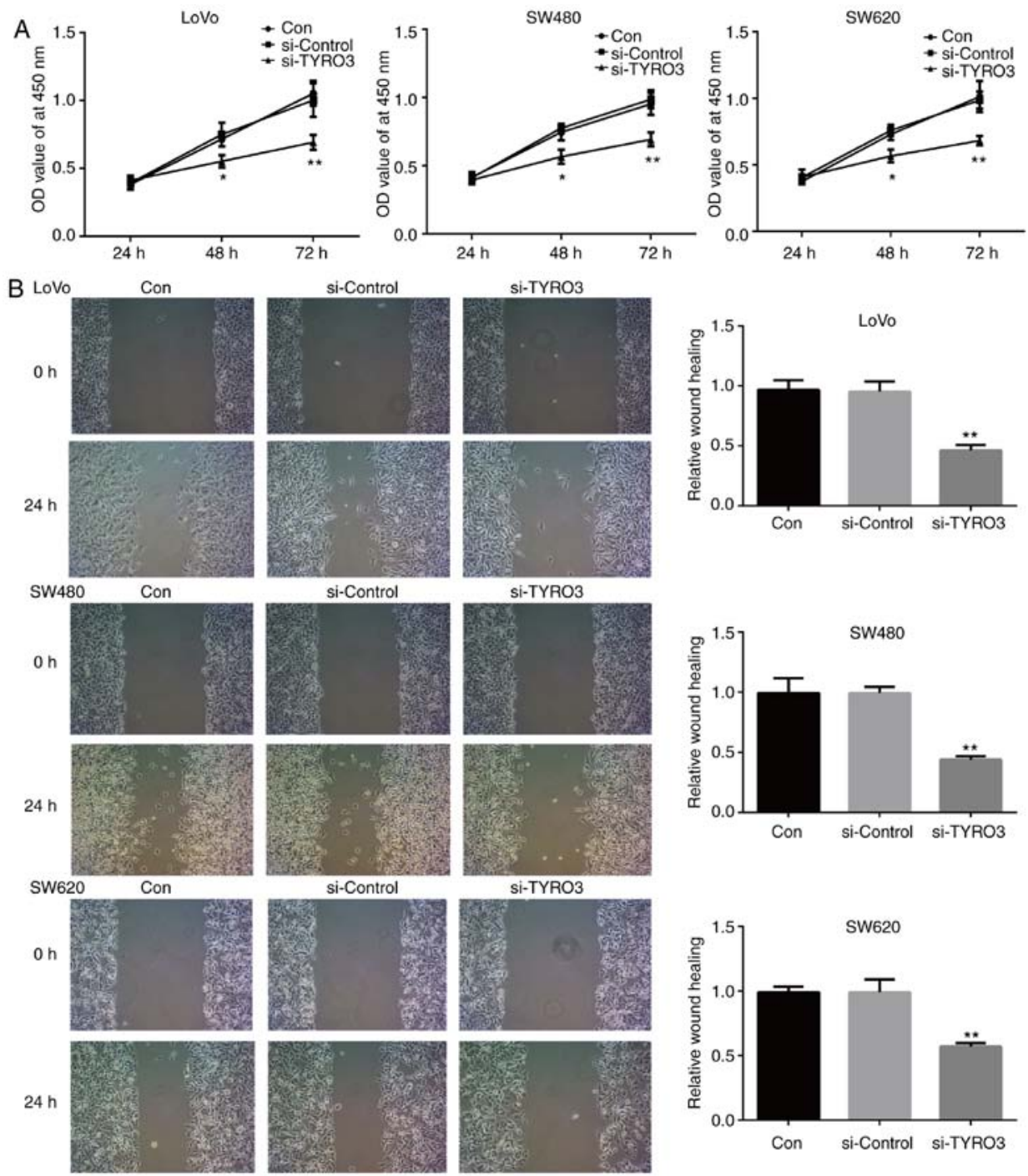

Figure 5. TYRO3 downregulation suppresses colorectal cancer cell proliferation and migration. LoVo, SW480 and SW620 cells were transfected with si-Control or si-TYRO3. (A) Effect of TYRO3 downregulation on the proliferation of LoVo, SW480 and SW620 cells, assessed by MTT assay. (B) Effect of TYRO3 downregulation on the migration of LoVo, SW480 and SW620 cells, assessed by a wound-healing assay. Data are expressed as the mean \pm standard deviation. ${ }^{*} \mathrm{P}<0.05$ and ${ }^{* *} \mathrm{P}<0.01$, vs. Con group. si-, small interfering RNA; Con, Control.
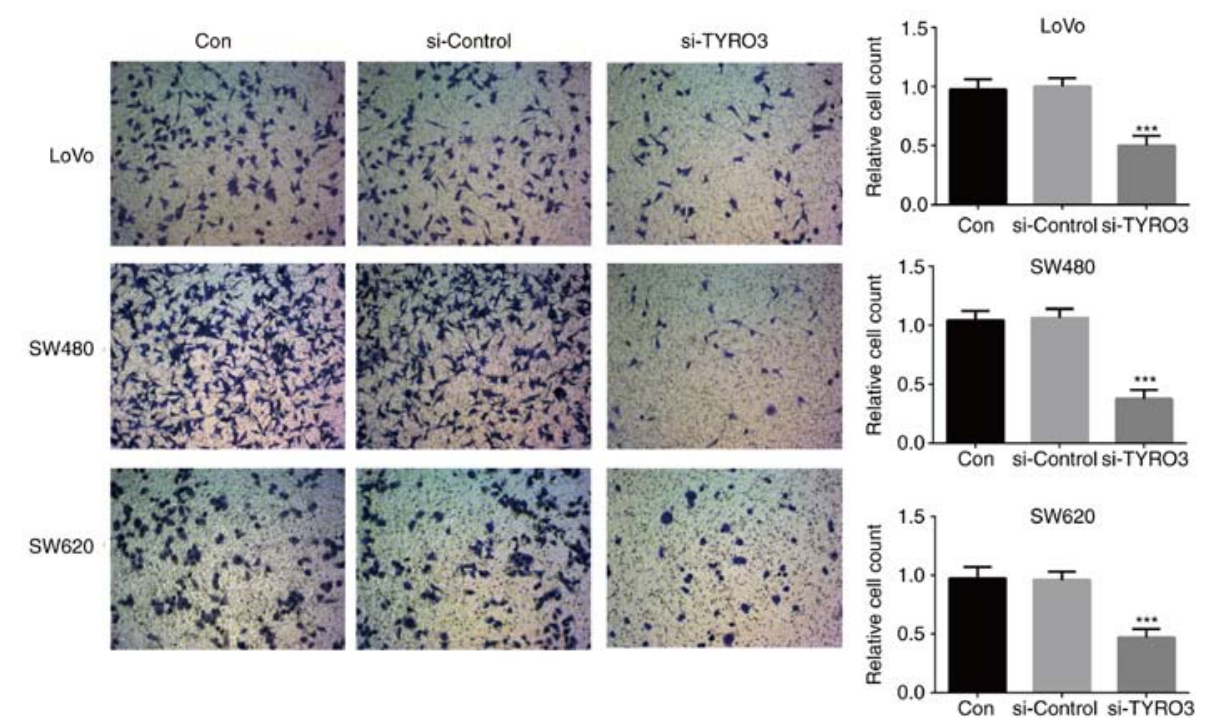

Figure 6. TYRO3 downregulation suppresses colorectal cancer cell invasion. Transwell assays were used to examine the invasion ability of LoVo, SW480 and SW620 cells transfected with si-Control or si-TYRO3. Data are expressed as the mean \pm standard deviation. ${ }^{* * * *} \mathrm{P}<0.001$ vs. Con group. si-, small interfering RNA; Con, Control. 

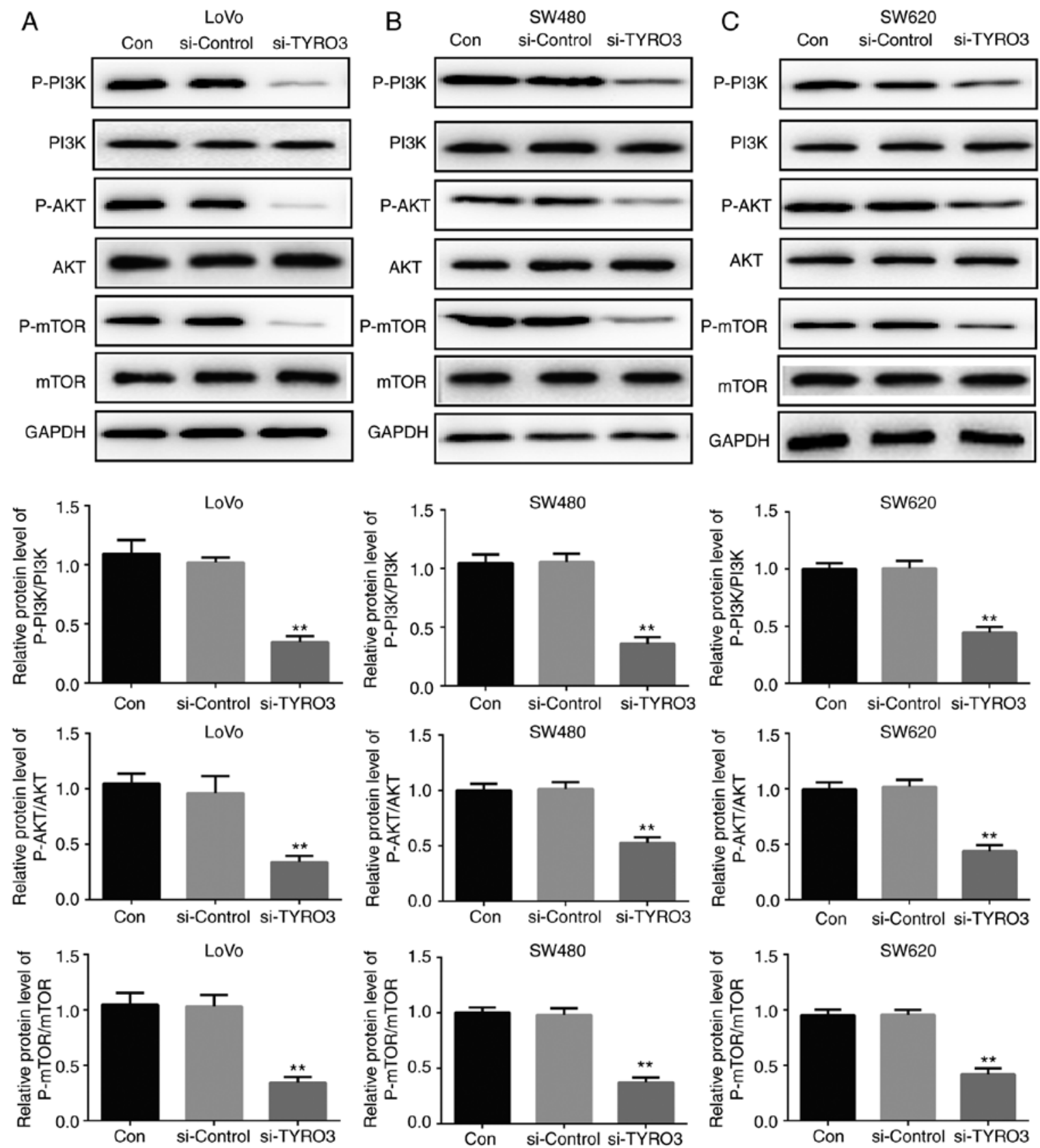

Figure 7. TYRO3 downregulation inhibited the activation of the PI3K/AKT/mTOR pathway in colorectal cancer cells. Western blot analysis was used to examine the protein expression levels of p-PI3K, PI3K, p-AKT, AKT, p-mTOR and mTOR in (A) LoVo, (B) SW480 and (C) SW620 cells transfected with si-Control or si-TYRO3. Data are expressed as the mean \pm standard deviation. ${ }^{* *} \mathrm{P}<0.01$, vs. Con group. si-, small interfering RNA; Con, Control; PI3K, phosphoinositide 3-kinase; AKT, protein kinase B; mTOR, mammalian target of rapamycin; p-, phosphorylated.

expression was also observed to be significantly associated with poor overall survival in patients with CRC $(\mathrm{P}=0.010)$, while miR-7 expression was an independent prognostic factor in these patients (hazard ratio, 1.854; $95 \%$ confidence interval, 1.016-3.540; $\mathrm{P}=0.044$ ) (21). Thus, miR-7 functions as an oncogene in CRC, and is significantly associated with tumor progression and poor prognosis. In addition, multivariate analysis indicated that low miR-7 expression was an independent prognostic factor for poor survival $(\mathrm{P}=0.0430)(10)$. miR-7 was reported to be downregulated in non-small cell lung cancer cell lines, and its overexpression induces cell apoptosis and suppresses cell proliferation, migration and tumorigenicity by targeting
B-cell lymphoma-2 (23). In addition, it has been reported that miR-7 was downregulated in breast cancer, and associated with epithelial-to-mesenchymal transition and metastasis (24). Furthermore, miR-7 inhibits CRC proliferation and induces apoptosis by targeting X-ray repair cross complementing 2 (22). A miR-7, as a novel miRNA with tumor suppressive function in CRC, was confirmed in nude mice to induce apoptosis through regulation of the early cell-cycle checkpoint (25). The present study demonstrated that miR-7 was downregulated in CRC cells, and that miR-7 overexpression inhibited CRC cell proliferation, invasion and angiogenesis, which was consistent with the findings of a previous study (25). 



Figure 8. miR-7 downregulation promoted the proliferation and invasion of colorectal cancer cells via directly targeting TYRO3, while TYRO3 repressed the function of miR-7 in these cells. (A) Western blot analysis was used to examine the protein expression levels of TYRO3 in LoVo, SW480 and SW620 cells transfected with NC or miR-7 inhibitor, or co-transfected with miR-7 inhibitor and si-TYRO3. (B) MTT and (C) Transwell assays were used to examine the cell proliferation and invasion, respectively, of LoVo, SW480 and SW620 cells in the different groups. Data are expressed as the mean \pm standard deviation. $\mathrm{P}<0.05$ and ${ }^{* *} \mathrm{P}<0.01$, vs. control groups; ${ }^{\# \#} \mathrm{P}<0.01$ and ${ }^{\# \#} \mathrm{P}<0.001$, vs. miR-7 inhibitor. Con, Control; NC, inhibitor control; si-TYRO3, TYRO3 small interfering RNA; miR, microRNA.

Bioinformatics analysis in the current study predicted that TYRO3 is a potential direct target of miR-7, which was also consistent with previous findings (16). It was thus suggested that miR-7 may function by interacting with TYRO3. To experimentally validate this hypothesis, the expression of TYRO3 in human CRC cells was initially examined. As expected, the results demonstrated that the levels of TYRO3 expression in CRC cells were elevated and inversely associated with miR-7 expression. Previous studies have reported that TYRO3 is overexpressed in patients with CRC, as well as in liver cancer metastases in comparison with normal liver $(13,26)$. Furthermore, TYRO3 has previously been identified as a novel 

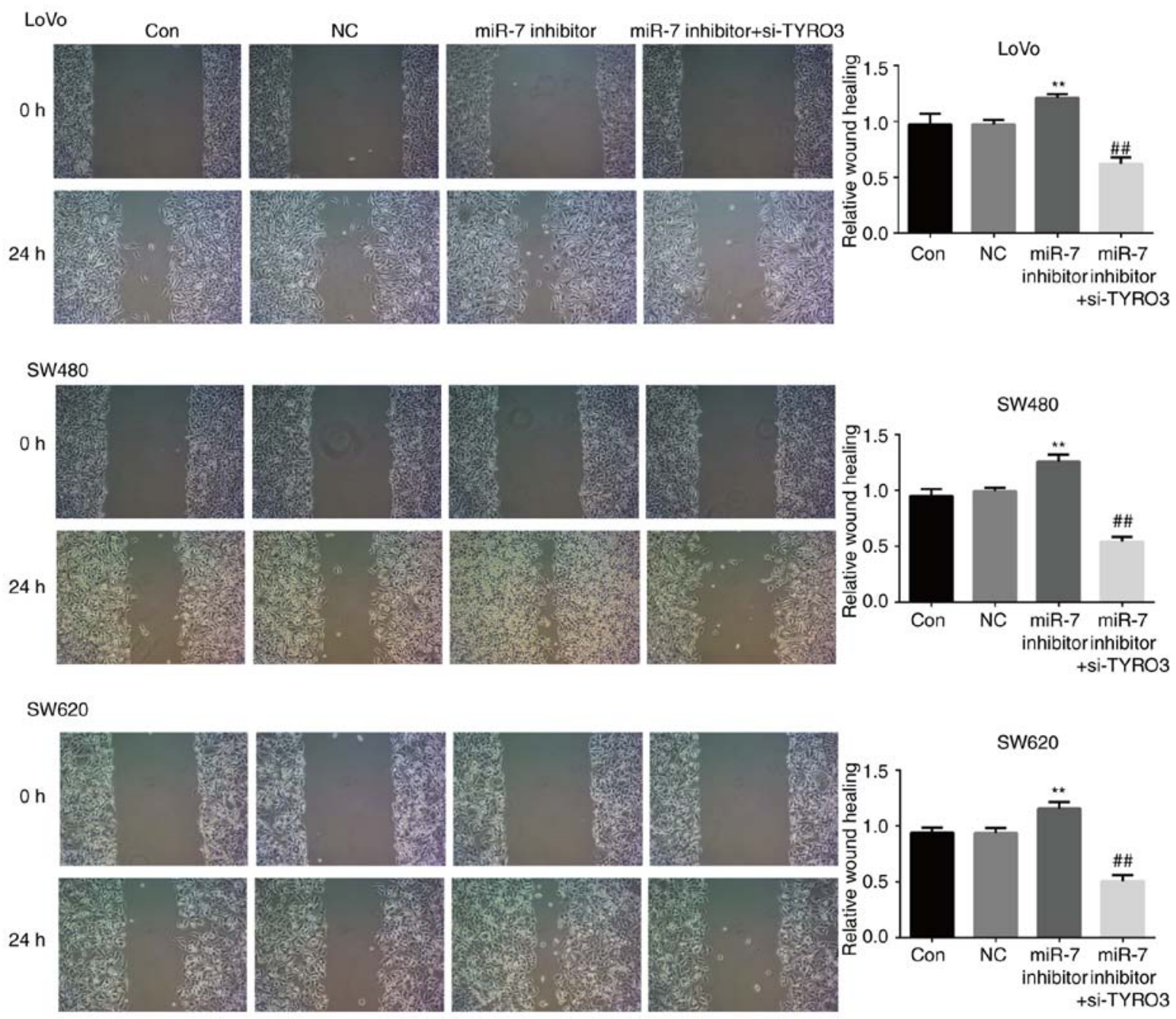

Figure 9. miR-7 downregulation promoted the migration of colorectal cancer cells via directly targeting TYRO3. Wound-healing assay was used to examine the migration of LoVo, SW480 and SW620 cells transfected with NC or miR-7 inhibitor, or co-transfected with miR-7 inhibitor and si-TYRO3. Data are expressed as the mean \pm standard deviation. ${ }^{* *} \mathrm{P}<0.01$, vs. control groups; ${ }^{* \#} \mathrm{P}<0.01$, vs. miR-7 inhibitor. Con, Control; NC, inhibitor control; si-TYRO3, TYRO3 small interfering RNA; miR, microRNA.

target of miR-7 in CRC (16). It has been revealed that high expression of TYRO3 in tumor tissues significantly reduced the survival of patients with CRC (13). In the present study, the results demonstrated that knockdown of TYRO3 with siRNA significantly inhibited the proliferation, migration and invasion capacity of CRC cells, indicating that TYRO3 expression was involved in CRC. However, whether miR-7 exerted its tumor suppressor effects in CRC by targeting TYRO3 has not been previously reported, to the best of our knowledge. Therefore, the present study is the first to demonstrate that TYRO3 repressed the inhibitory effects of miR-7 on CRC cell proliferation, migration and invasion.

A previous study has reported that TYRO3 modulates several oncogenic pathways, including those involved in survival, altered cellular morphology, cell cycle transition, proliferation, adhesion and motility (27). In addition, it has previously been observed that TYRO3 binds to growth-inhibitory specific 6 receptors through the PI3K/AKT/mTOR or RAF/MEK/ERK1/2 pathways in order to control cell proliferation and survival $(28,29)$. TYRO3 has also been identified as a key driver of the oncogenic PI3K/AKT pathway in hepatocellular carcinoma (30). However, it remained unclear whether miR-7 functions through interaction with the TYRO3-dependent PI3K/AKT/mTOR signaling pathway. In the present report, the results revealed that the inhibition of miR-7 markedly increased the activity of PI3K/AKT signaling pathway in human CRC cell lines, while this effect was inhibited by TYRO3 siRNA. Therefore, the present study clarified a novel mechanism of miR-7 in regulating the proliferation, migration and invasion of CRC cells. Future studies will aim to confirm this mechanism using in vivo models.

In conclusion, the present study demonstrated that miR-7 inhibited the proliferation, migration and invasion of CRC cell lines via direct inhibition of TYRO3, which may be mediated by the PI3K/AKT/mTOR signaling pathway. This suggested that TYRO3 and miR-7 may serve as promising diagnostic and therapeutic targets in CRC.

\section{Acknowledgements}

The authors would like to thank Dr Yu Luo and Dr Hai Huang (Department of General Surgery, The Affiliated Suzhou Hospital to Nanjing Medical University) for collecting the data reported in the article. 



Figure 10. miR-7 downregulation promoted the activation of PI3K/AKT/mTOR pathway via directly targeting TYRO3 in colorectal cancer cells. Western blot analysis was used to examine the protein expression levels of p-PI3K, PI3K, p-AKT, AKT, p-mTOR and mTOR in (A) LoVo, (B) SW480 and (C) SW620 cells transfected with NC or miR-7 inhibitor, or co-transfected with miR-7 inhibitor and si-TYRO3. Data are expressed as the mean \pm standard deviation. ${ }^{* *} \mathrm{P}<0.01$, vs. control groups; ${ }^{\# \# ~} \mathrm{P}<0.01$, vs. miR-7 inhibitor. miR, microRNA; PI3K, phosphoinositide 3-kinase; AKT, protein kinase B; mTOR, mammalian target of rapamycin; p-, phosphorylated; Con, Control; NC, inhibitor control; si-TYRO3, TYRO3 small interfering RNA.

\section{Funding}

No funding was received.

\section{Availability of data and materials}

The analyzed data sets generated during the present study are available from the corresponding author on reasonable request.

\section{Authors' contributions}

AQ wrote the manuscript and analyzed and interpreted the data. WQ designed the study and revised the manuscript. Both authors read and approved the final manuscript.

\section{Ethics approval and consent to participate}

Each patient provided written informed consent, and all experimental protocols were approved by the Ethics Committee of the Affiliated Suzhou Hospital of Nanjing Medical University (Suzhou, China).

\section{Patient consent for publication}

Not applicable.

\section{Competing interests}

The authors declare that they have no competing interests. 


\section{References}

1. Siegel RL, Miller KD and Jemal A: Cancer statistics, 2018. CA Cancer J Clin 68: 7-30, 2018.

2. Ferlay J, Soerjomataram I, Dikshit R, Eser S, Mathers C, Rebelo M, Parkin DM, Forman D and Bray F: Cancer incidence and mortality worldwide: Sources, methods and major patterns in GLOBOCAN 2012. Int J Cancer 136: E359-E386, 2015.

3. Van Cutsem E, Cervantes A, Nordlinger B and Arnold D; ESMO Guidelines Working Group: Metastatic colorectal cancer: ESMO Clinical Practice Guidelines for diagnosis, treatment and follow-up. Ann Oncol 25 (Suppl 3): iii1-iii9, 2014.

4. Siegel R, Desantis C and Jemal A: Colorectal cancer statistics, 2014. CA Cancer J Clin 64: 104-117, 2014.

5. Bartel DP: MicroRNAs: Genomics, biogenesis, mechanism, and function. Cell 116: 281-297, 2004.

6. Petri A, Lindow M and Kauppinen S: MicroRNA silencing in primates: Towards development of novel therapeutics. Cancer Res 69: 393-395, 2009.

7. Landi MT, Zhao Y, Rotunno M, Koshiol J, Liu H, Bergen AW, Rubagotti M, Goldstein AM, Linnoila I, Marincola FM, et al: MicroRNA expression differentiates histology and predicts survival of lung cancer. Clin Cancer Res 16: 430-441, 2010.

8. Loo JM, Scherl A, Nguyen A, Man FY, Weinberg E, Zeng Z, Saltz L, Paty PB and Tavazoie SF: Extracellular metabolic energetics can promote cancer progression. Cell 160: 393-406, 2015.

9. Chen WQ, Hu L, Chen GX and Deng HX: Role of microRNA-7 in digestive system malignancy. World J Gastrointest Oncol 8: 121-127, 2016

10. Suto T, Yokobori T, Yajima R, Morita H, Fujii T, Yamaguchi S, Altan B, Tsutsumi S, Asao T and Kuwano H: MicroRNA-7 expression in colorectal cancer is associated with poor prognosis and regulates cetuximab sensitivity via EGFR regulation. Carcinogenesis 36: 338-345, 2015.

11. Li Y, Li Y, Liu Y, Xie P, Li F and Li G: PAX6, a novel target of microRNA-7, promotes cellular proliferation and invasion in human colorectal cancer cells. Dig Dis Sci 59: 598-606, 2014.

12. Ohashi K, Mizuno K, Kuma K, Miyata T and Nakamura T: Cloning of the cDNA for a novel receptor tyrosine kinase, Sky, predominantly expressed in brain. Oncogene 9: 699-705, 1994.

13. Schmitz R, Valls AF, Yerbes R, von Richter S, Kahlert C, Loges S, Weitz J, Schneider M, Ruiz de Almodovar C, Ulrich A and Schmidt T: TAM receptors Tyro 3 and Mer as novel targets in colorectal cancer. Oncotarget 7: 56355-56370, 2016.

14. Vouri M and Hafizi S: TAM receptor tyrosine kinases in cancer drug resistance. Cancer Res 77: 2775-2778, 2017

15. Martinelli E, Martini G, Cardone C, Troiani T, Liguori G, Vitagliano D, Napolitano S, Morgillo F, Rinaldi B, Melillo RM, et al: AXL is an oncotarget in human colorectal cancer. Oncotarget 6: 23281-23296, 2015.

16. Kabir TD, Ganda C, Brown RM, Beveridge DJ, Richardson KL, Chaturvedi V, Candy P, Epis M, Wintle L, Kalinowski F, et al: A microRNA-7/growth arrest specific 6/TYRO3 axis regulates the growth and invasiveness of sorafenib-resistant cells in human hepatocellular carcinoma. Hepatology 67: 216-231, 2018.

17. Engelman JA, Luo J and Cantley LC: The evolution of phosphatidylinositol 3-kinases as regulators of growth and metabolism. Nat Rev Genet 7: 606-619, 2006.
18. Chaturvedi MM, Sung B, Yadav VR, Kannappan R and Aggarwal BB: NF- $\kappa \mathrm{B}$ addiction and its role in cancer: 'One size does not fit all'. Oncogene 30: 1615-1630, 2011.

19. Thorson AG, Christensen MA and Davis SJ: The role of colonoscopy in the assessment of patients with colorectal cancer. Dis Colon Rectum 29: 306-311, 1986.

20. Pfaffl MW: A new mathematical model for relative quantification in real-time RT-PCR. Nucleic Acids Res 29: e45; 2001.

21. Nagano Y, Toiyama Y, Okugawa Y, Imaoka H, Fujikawa H, Yasuda H, Yoshiyama S, Hiro J, Kobayashi M, Ohi M, et al: MicroRNA-7 is associated with malignant potential and poor prognosis in human colorectal cancer. Anticancer Res 36: 6521-6526, 2016.

22. Xu K, Chen Z, Qin C and Song X: miR-7 inhibits colorectal cancer cell proliferation and induces apoptosis by targeting XRCC2. Onco Targets Ther 7: 325-332, 2014.

23. Xiong S, Zheng Y, Jiang P, Liu R, Liu X and Chu Y: MicroRNA-7 inhibits the growth of human non-small cell lung cancer A549 cells through targeting BCL-2. Int J Biol Sci 7: 805-814, 2011.

24. Zhang H, Cai K, Wang J, Wang X, Cheng K, Shi F, Jiang L, Zhang Y and Dou J: MiR-7, inhibited indirectly by lincRNA HOTAIR, directly inhibits SETDB1 and reverses the EMT of breast cancer stem cells by downregulating the STAT3 pathway. Stem Cells 32: 2858-2868, 2014.

25. Zhang N, Li X, Wu CW, Dong Y, Cai M, Mok MT, Wang H, Chen J, Ng SS, Chen M, et al: microRNA-7 is a novel inhibitor of YY1 contributing to colorectal tumorigenesis. Oncogene 32: 5078-5088, 2013.

26. Chien CW, Hou PC, Wu HC, Chang YL, Lin SC, Lin SC, Lin BW, Lee JC, Chang YJ, Sun HS and Tsai SJ: Targeting TYRO3 inhibits epithelial-mesenchymal transition and increases drug sensitivity in colon cancer. Oncogene 35: 5872-5881, 2016.

27. Zhu S, Wurdak H, Wang Y, Galkin A, Tao H, Li J, Lyssiotis CA, Yan F, Tu BP, Miraglia L, et al: A genomic screen identifies TYRO3 as a MITF regulator in melanoma. Proc Natl Acad Sci USA 106: 17025-17030, 2009.

28. Gustafsson A, Boström AK, Ljungberg B, Axelson $\mathrm{H}$ and Dahlback B: Gas6 and the receptor tyrosine kinase Axl in clear cell renal cell carcinoma. PLoS One 4: e7575, 2009.

29. Ou WB, Corson JM, Flynn DL, Lu WP, Wise SC, Bueno R, Sugarbaker DJ and Fletcher JA: AXL regulates mesothelioma proliferation and invasiveness. Oncogene 30: 1643-1652, 2011.

30. Zhong Z, Wang Y, Guo H, Sagare A, Fernández JA, Bell RD, Barrett TM, Griffin JH, Freeman RS and Zlokovic BV: Protein $S$ protects neurons from excitotoxic injury by activating the TAM receptorTyro3-phosphatidylinositol3-kinase-Aktpathwaythrough its sex hormone-binding globulin-like region. J Neuroscience 30: 15521-15534, 2010.

This work is licensed under a Creative Commons Attribution-NonCommercial-NoDerivatives 4.0 International (CC BY-NC-ND 4.0) License. 